\title{
USING AUTOMATION TECHNOLOGY AND IOT BASED DATA CAPTURING TO ENSURE HIGH QUALITY LAST MILE LOGISTICS
}

\author{
Paras Arora, Swadesh Srivastava and Snigdha Majumder \\ Flipkart Internet Private Ltd \\ Bangalore, Karnataka 560034, India
}

\begin{abstract}
The customer facing leg, i.e. the Last Mile (LM), and field executive (FE) for any logistics company play a pivotal role in establishing a brand among its customers. It is imperative to have a robust and closely controlledLM for longevity of a company in the market.In the following paper, we present various sense-\&-respond concepts and technologies that can be used to track and, in-turn, enhance the performance of individual LM resources. Sense- \&-respond systems can lead to welldefined action plan enabling an efficient LMservice, thus improving customer experience, curtailing unnecessary costs and improving safety of all stakeholders. The different modular and customizable solutions conceptualized and evaluated below include multiple IOT technologies, like camera based solutions, BLE beacons, GPS, and recorders, that can be employed to have a multi-point sensing in LMdelivery service. We cover aspects, ranging from FE attendance, FE's disposition, track and trace of FEs in turn shipments, and route optimization, customers' and FEs' interaction and a feedback mechanism to allow for continuous improvement of customers' and FEs' experience.
\end{abstract}

Keywords: Last Mile (LM), Field Executive (FE), IOT, Sense-\&-Respond

\section{Introduction}

Last Mile(LM) is the final leg in logistics, delivering the goods to the customer. The forward part starts from the local distribution centres and culminates at the customers' doorstep. There is also reverse logistics of picking up shipments from customers and dropping at the distribution centres for further processing. Due to factors like traffic congestion,multiple drop points, and low shipment density per customer, LM is often the most expensive(as shown in Fig 1.) and least efficient link in the supply chain, yet one of the most crucial as there is direct interactionwith the customer. Any customer impression made in LM by the service or the interaction with the FE can impact future business from the particular customer. Having a swift, reliable, and customer friendly, yet cost effective LM service is critical for any logistics company looking for long term sustainability.

Morgan Stanley estimates the e-commerce market, in India, to grow over $\$ 100$ Billion by $2020^{[2]}$, with around $30 \%$ share of e-retailers, and over 300 million users. In the frenzy to capture the largest market share, most e-commerce companies are offering heavy discounts, leading to losses.To curb these losses, companies cut down operational costs. Customer experience centric initiatives,seem as additional costs in the short term, are often relativelyneglected in suchlogistics companies.

It should be remembered that a firm may be cost efficient by optimally using its resources in producing a given mix of outputs. Despite being cost efficient, this firm may not realize maximum possible profits if it fails to estimate market demand correctly and, thus, produces outputs that do not effectively match customer needs (Krasnikov, Jayachand-ran, \& Kumar, 2009 ${ }^{[3]}$ ). To address this issue, profit efficiency was introduced as a more inclusive concept than cost efficiency (Berger, Cummins, and Weiss $1995^{[4]}$ ). Profit efficiency focuses on unobserved differences in the extent to which the output of different firms meets customer needs, and it accounts for the notion that some firms may incur additional costs in providing superior services and products but are rewarded for these efforts through higher revenues. In effect, the profit efficiency concept captures the cost of inputs required to produce a certain level of outputs and the additional revenues generated by producing outputs that are best suited to meeting customer needs. Following the same philosophy, proposed IOT based sense-and-respond methods will require cost investment by the firms but will over-time incentivise the firms by maximising their profits, through greater customer satisfaction and retention, 
and minimizing their losses, by enabling better trace and track capabilities.

SinceLM delivery is a manual-labour intensive process, with FEs picking and dropping shipments betweendistributioncentres and customers, it brings along multiple inefficacies and shrinkages accrued with manual processes. In developing economies, unstructured manpower accentuates theft related problems such as theft and intentional mistakes.WithIOT based sense-and-respond systems, we can monitor the entire LM activity and material flow, and actively take corrective actions to maintain a strong LM service.Via this paper, we propose technologies to monitor and improve
Studies point that LM employees' performance can increase not only brand value but also regain/improve customer trust in the brand (Kuehner-Herbert, 2009 ${ }^{[5]}$; Ind, 2004 ${ }^{[6]}$ ), thus ensuring FEs' code of conduct is a priority for companies. Thompson and Maoz, 2005 ${ }^{[7]}$ suggest that $70 \%$ of spending for customer relationship in the coming years will be justified by its potential to increase efficiency. Customer specific initiatives requires additional resources. Profit efficient initiatives although, generate revenue that exceeds the additional costs. In effect, such initiatives enable firms to generate higher-quality products and services, possibly at higher costs. The proposed technologies, among other existing methods,

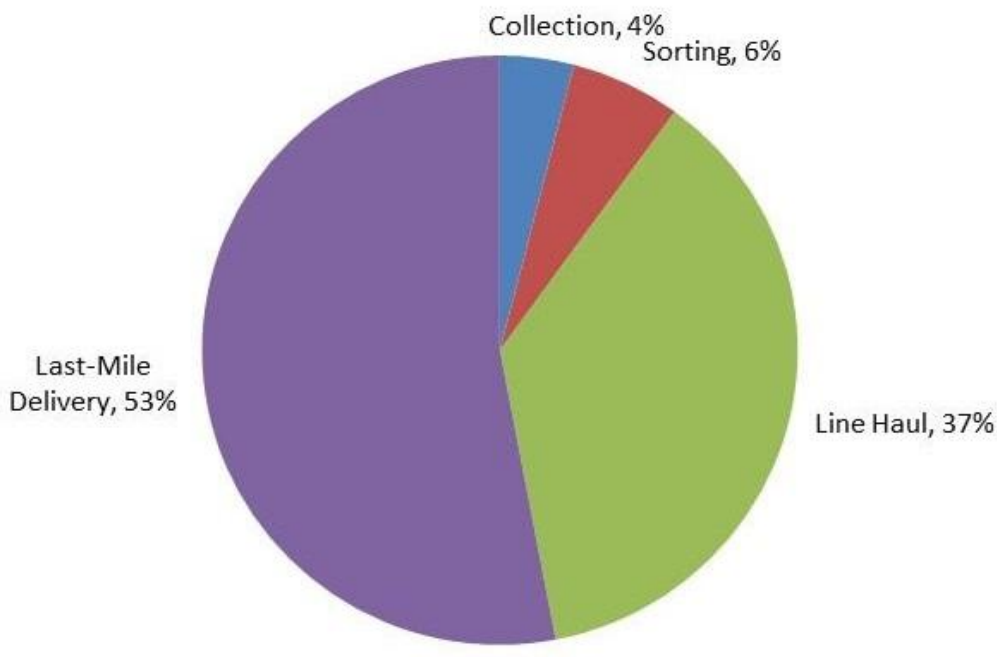

Collection Sorting Line Haul Last-Mile Delivery

Figure 1. Industry wide cost breakup of logistics companies (Millar, 2005) ${ }^{[1]}$

productivity of FEs, reduce misconduct, decrease FE related escalations, and improve FEs and customers' safety.In the next section, we realize what are some of the current metrics for the large players in the supply chain industry, focussed on Indian markets, and in the following section possible solutions are offered that may be employed to improve these metrics.

\section{Customer Relationship - The elusive challenge}

"One thing Amazon [U.S.] has done very successfully [is that] they've owned the entire value chain. They've owned the last mile, the moment that matters - when the package arrives. Once you can own [that], you build a loyal customer base." - SucharitaMulpuru, Forrester Research facilitate efficient information flow between a firm and its customers by capturing and routing of relevant information to appropriate channels. Firms that have better relationships with customers enjoy higher profitability (e.g., Bolton $1998^{[8]}$; Reinartz, Thomas, and Kumar $2005^{[9]}$ ). With companies that maintains and executes better communication with their customers, their customers are likely to stay longer in the relationship with them, purchase more often, and show lower propensity to switch to competitors (Johnson and Selnes 2004 ${ }^{[10]}$ ).

In developing economies like that of India, ecommerce is in a nascent stage, majorly due to reduced internet reach and the legacy of brick and mortar stores that offer a personal connect. Tangibility of the items in offline stored allows customers to experience the items before purchasing them. Due to this factor, online stores 
had to come up with lenient return policies and cash-on-delivery (COD) option. Currently around $80 \%$ of customers opt for COD. It leads to slower delivery service as each transaction takes extra time to complete. Companies set up customer care centers to guide customers and assay the escalations that could relate to FEs' attitude or product errors. A reliable delivery could provide confidence to customers to opt for pre-payment and curb call center costs.

In this study, we have targeted improving customer relationship through provision of efficient and reliable delivery, along with measures towards safety of both participants, i.e. FEs and customers.

\section{Customer Experience}

Withincreasing urbanization, changing customer priorities and propensity to spend, and increasing number of competitors, customer experience can prove to be a differentiator for companies wanting to stay in the business for long. Moreover, it can be a marketing tool through word of mouth and ways as described later. As per Cadwallader et al, $2010^{[11]}$, customer perception of a brand depends on front line employees/administration behaviour, and attitude.

Given the large expanse of India and high population density, companies often prefer to locate distribution centers as close to the customers as possible. This is to limit customer per FE, hence, speedier and relatively easier for managing orders. Flip-side of such a highly distributed network is that since there are innumerable employees in the LM of a large logistics company, it is laborious and next to impossible to maintain a standard quality of operations through-out the network. Multiple firms have close-to-customer hubs or service centres with person in-charge to monitor $10-50$ people under him / her. Beyond a certain configuration, it can be an arduous task for the hub in-charge and often leads to unintended lax or oversights. Moreover, on-the-field monitoring of FE's performance remains an almost impossible challenge to tackle. Through multiple surveys, scalations related to FE generally fall under:

- Unprofessional behaviour and dress

- $\quad$ Asking for tip

- $\quad$ FE denies coming to doorstep

- Delivers to wrong person (eg. security or relative) and does not record the same

Currently there is no evidence in case for conflict resolution. As it will be made clear in the following sections, technology can put eyes and ears on site and provide visibility through the entire LM leg.

\section{Reliable Delivery}

E-commerce thrives on convenience of shopping and getting items delivered at doorstep with just a few clicks. An unreliable LM service, with uncertainties in arrival time, making the customer wait or even pursue the delivery service can cause the e-retailers to lose customers. A reliable delivery service on the other hand offers tracking of the shipments, as well as, at the next level, assures slotted delivery, i.e. within the time slot decided with the customer.

Currently it takes on an average 6-7 days by Indian e-commerce companies (4-6 days for Flipkart shipments) [Red Seer Consulting report, 2016]. For most services, customer is unaware as to when the shipment is planned to arrive within the day and might not be available when the FE arrives. Besides, often the customer, due to either ignorance or intent, enters wrong pin codes in the address, which throws off the delivery service. The inability to perfectly coordinate with the FE and erroneous address details, leads to, at best, less productive LM, and, at worst, multiple failed attempts and even return of shipments that costs a company, across India e-commerce industry, directly around Rs. 49 per attempt [Red Seer Consulting report, 2016]. Add to that indirect costs such as customer escalations, for which a customer care must be setup, and reduction of reorders.

A major contributor to the cost, and companies' troubles is failed and invalid attempts. As per various articles published in reputed newspapers, percentage of failed attempts can be between 15$20 \%$ in major cities and, even $30-40 \%$ in smaller towns ${ }^{[12,13,14]}$. IOT can help track FEs and in verifying the customer $-\mathrm{FE}$ interactions.

\section{Safety}

In multiple recent incidences, safety concerns have been raised for customers and even FEs (as they are put to harm's way if someone tries to steal the merchandize $\left.\mathrm{i}^{15,16,17}\right)$. Once again companies feel help -less when it comes to tracking the event or collecting evidences.

Unwarranted delays, suspicious interactions and dubious transactions, can be recorded and monitored with IOT devices. They can assist in developing the back-end data analytics to 
proactively prevent any undesired incidences and alleviate the concerns.

\section{Technology to the aid}

Information drives decisions and policies. Without extensive data, any policy is merely a shot in the dark. Internet of things, through omnipresent network of sensors, bridges these gaps, connecting the entire network. The explosion of connected devices combined with central processingecommerce platforms and adoption of common standards will only intensify the growth of IoT-enabled capabilities across the retail industry. Various sensors and systems can be employed to effectuate a reliable and customer centric LM logistics. Following list explores such sense-andrespond systems, through the LM delivery,focusingon the human element.Shipment tracking techno-logy such as RFID etc.

\section{Vision based Attendance and Grooming Check}

Cognizance of the attendance for the day is a basic requirement for any labour intensive company and is needed for a reliable delivery service. In LM, it allows planning the routes and responsibility for the FEs and disbursing salaries correctly. Often companies use either fingerprint based biometric machines, card readers or record it manually. Most reliable of course is biometric machine.

"Good grooming is integral and impeccable style is a must. If you don't look the part, no one will want to give you time or money." - Daymond John

In an internal survey, around 50\% customers considered FE grooming as an important factor for perfect delivery, as it establishes trust on the FE. The FE for any company is not just some service or delivery personnel but is the customer facing brand ambassador. A positive demeanour and grooming inspires trust and respect for the person and the company tag he / she carries. Realizing this fact, companies often set grooming standards and uniforms to FEs.

Flipkart, like many other customer facing logistics companies, defines grooming parameters for the FEs. The norms encompass FE t-shirt, jacket, facial hair, and shoes, for a professionalappearance.Accordingly, uniforms are provided to the FEs that project the brand. To ensure compliance, team leader appraises the FEs before assigning them tasks. It can take considerable amount of time (1-2 min per FE) to complete the associated task-list, and record data. Team leadersalso have considerable responsibility to control the LM of his / her area, Thus, due to lack of time or negligence, grooming check takes a back seat, leading to erroneous data.

To expedite the process, automated vision based attendance and grooming is being piloted at Flipkart distribution hubs where an FE is identified and his / her grooming score is generated based on the above-mentioned parameters. The scores are linked with the FE's identity, in the ERP. Aside from savings on the biometric machines (which are often expensive), it can help reduce the operational costs by up to $10-12 \%$ per team (depending on the team size) a month.

Multiple options were explored to take the photos, using fixed cameras of different resolutions as well as mobile cameras. Each option entails associated complexities. Pilot yielded $100 \%$ accuracy in facial recognition and dress code check, and based on the chosen method, 70\% - 99.8\%accuracy for facial hair recognition(roof mounted $2 \mathrm{MP}$ camera being the least accurate, and selfie being the highest). The selfie basedsolution is easily scalable and has an expected payback period of 9 months.

\section{GPS}

All deliveries happen on either bikes or vans. GPS capability in the vehicles offers irrefutable benefits in logistics via asset tracking. GPS tracking helps in improving all three fronts - reliability, safety and customer experience thanks to following potentials:

1. Route planning and monitoring: First and foremost,mapping and en-route asset visibility allows real time route planning. This is exceptionally useful for short delivery services like food delivery, and multiple concierge services. Business model of cab services like Ola and Uber thrives on GPS enablement. Moreover, any deviations can be monitored and flagged. The outliers can be identified and respective actions can be initiated.

2. Fleet management: Real time positioning, allows coordinating FEs on road. Thus, re-routing, and task sharing is made possible by having GPS capability.

3. Estimate time of delivery\& real time updates: Based on route planning and current position of the $\mathrm{FE}$, prior intimations can be made to the customer and route can be re-planned in case of any issue. Customer can be ready in case on delivery or free 
his/her schedule accordingly. This saves customers', as well as, FEs' time, hence positively impacts customer experience and FE productivity.

4. Fuel consumption: Fuel cost is a high contributor to total LM cost. Payments are often made per $\mathrm{km}$. It has been observed that many vehicle owners manipulate odometers to display more than actual distances, by as much as $20 \%$. Any such deviations can be tracked, and expected fuel consumption on the route can be compared with actual to reckon any fuel theft as well as control costs.

5. Address verification: In multiple incidences, customers intentionally,erroneously, or by ignorance, or enter wrong addresses or pin codes and call up the FE to follow a different route. This impacts FEs performance, and the following route and deliveries, hence adversely affecting the customer experience. By event based GPS pings, exact delivery locations can be marked for future reference and even analysing customer behaviour.

6. Vehicle and driver safety: As per Ministry of Road Transport \& Highway, a serious road accident occurs every minute in India. Through real time visibilityany events like over-speedingor unexpected delays can be tracked.Driving patterns can be recorded and appropriate action taken. It even enables theft capturing, of shipments (as for unexpected stops), and the vehicle.This can lead to $5-15 \%$ saving of insurance costs ${ }^{[18]}$.

7. Evidence in case of delivery disputes: Escalations like False Attempts (e.g. FE did not arrive, customer absent etc.) can be resolved.

GPS capabilities and back end analytics can impact multiple aspects of transport logistics and are elevating from a luxury to a necessity. These capabilities positivelyimpactLM planning, customer experience, maintaining FE performance, andotherindirect cost savings.

Considering the many benefits, most of the logistics companies across the world have already employed GPS in their heavy vehicles. GPS capabilities have been tested and phase wise implementation is under progress in Flipkart. First phase was leveraging the GPS capabilities of FE mobile phones that has LM app. For regular delivery service, GPS ping is triggered during important events such as deliveries. For short pick\&-drop services such as food delivery, regular every minute ping was enabled, to allow complete route visibility to the customers.
Due to limited batteries, mobile devices, although, cannot be used for high accuracy GPS pings for continuous 8hours of operation. Pilots conducted showed a regular mobile phone can last about 6-7 hours at high accuracy GPS phone, with the most basic apps running. The duration gets reduced as the number of apps increase and the phones get older. As per tests, the accuracy of mobile phone GPS can range between $30 \mathrm{~m}-50 \mathrm{~m}$ on road. This encouraged us to test a standalone pocket GPS, with a long battery and 10m accuracy. Such GPS devices can be obtained off-the shelf at low cost and give an offline download of the entire route taken be the FE. Costs increase if GPRS is desired for live information.

Certainly, it is desirableto have asset free solution, that does not add any discomfort to the FE, but allows constant and real time information at high accuracy. This encouraged us to explore en-route charging option, as detailed under:

\section{On the go Charging}

Given the shift from paper based deliveries, to smart device based deliveries, with route-mapping, delivery schedules, taking signatures with time stamps etc, it is desired that the FE be connected all the time and his device can perform throughout the working hours. With multiple sensors like GPS (as mentioned above), BLE, accelerometers etc, it is becoming increasingly difficult to sustain the battery for such durations. This raises a requirement of either additional power banks or a method to keep the devices live. Power-banks, although, have limit-ations such as limited number of charging cycles and possible oversight in charging by the FE.

Almost $80 \%$ of the LM deliveries, in India, happen via motor bikes. Moreover, these bikes are owned by the FEs themselves. This pushes for the requirement of easily pluggable devices on the bikes

1. Battery adaptor:One of the possible solutions is a USB charger connected to the bike battery. Such economical chargers can be purchased easily.

2. Dynamo: In an internal survey, it was realized that many FEs were uncomfortable in withdrawing extra connections from their bike batteries. This led us to explore another economic yet reliable method to charge phones during movement, a bike mounted dynamo that converts rotational energy of wheels or the transmission system in the bike into electrical energy to charge the phone. Well- 
designed dynamos can be easily mounted on the bikes before the shift and removed right after.

There are multiple variants available in the market for dynamos. A control circuit has been designed to use dynamos for charging phones and the entire system under exploration and tests. The hardware costslower than INR 300 and is easy to maintain.

\section{$B L E$}

GPS cannot be used in indoor environments, and, as mentioned, is battery intensive. In such cases, BLE technology can be leveraged as a location signature for a building or location, to maintain a reliable LM. This is especially useful for short distance pick and drop, concierge and food deliveries where the FE must confirm his presence at the location.

BLE beacons' range varies between 10-12 m and distance between receiver and emitter can be computed using RSSI (Recieved Signal Strength Indicator) data. Due to this precision, BLE is being used by multiple retail stores across the world for area specific advertisements and discounts to shoppers. Moreover, they are used for localization of assets within the facility.

Today every delivery person carries a smart phone with Bluetooth connectivity. Inexpensive BLE beacons of around INR 700 can be installed at the vendors' locations and relevant information and orders can be pushed to the FE and the network, even if $\mathrm{FE}$ is unable to access internet on mobile device. This helps in optimizing information sharing and elimination of fake attempts and claims by FEs and vendors.Additionally, the beacons can be used to push advertisements and offers for the ecommerce sites or vendors to customers in vicinity of beacons, thus bring additional dimension of marketing and thusproviding an alternate revenue source.

\section{Voice Recorder}

In allcustomer - FE interaction related escalations, companies find themselves at the receiving end, as the error or misconduct cannot be attributed to either the FE, or the customer. Moreover, in case of any safety concern or a crime, it becomes difficult to identify the wrong doer due to lack of evidence.

It is a regular practice to record call centre calls for training purposes and more. Similarly, a recording device with the FE that monitors the conversations and can raise flags during conversations can help identify unpleasant situations and provide evidence in case of any conflict. For handling large data, voice analytics software is used to identify tonalities, pitch, keywords etc and flag relevant conversations for further analysis. If implemented, multiple issues can be solved by monitoring the field conversations:

1. Reduction of Customer Escalations and improved CSAT (Customer SATisfaction) score: By setting conversational standards and using the recordings for training, customer-FE interaction can be made more professional and pleasant for the customers.

2. Shrinkage Capture: By analysing GPS data and recordings in tandem, point of theft and culprits involved can be identified. Through legal action, items may also be retrieved.

3. Customer retention: A customer happy with the service, who got the items he received as good condition, is more likely to re-order from the website

4. Safety of the FEs: If the recordings are being analysed in real time, unpleasant incidences can be identified as they happen. This can provide just enough time to take appropriate action.

5. Reduction in call centre costs: With reduced number of escalations, call centres can be offloaded.

6. Incident evidences - Legal advantages: In case of an unpleasant incident, ranging from arguments to safety concerns, recording of the incident can help implicate the culprit and take due action.

Legal approvals and customer consent must be considered before recording the conversations. We at Flipkart are currently exploring the legal implications of voice recorders thatwill be handled by local delivery centres.

\section{Smart Lockers}

Considering customers' privacy, unavailability of customers at mutually convenient time, and ease of package drops and retrievals, lockers are becoming a sensation in LM across the industry. They allow timely and assured delivery, affecting reliability and customer experience. Lockers can strategically be placed at various locations like shopping centres, metro and bus stations, grocery stores etc. and payment gateways can be enabled to facilitate even COD services.Customers can take the items at their convenience and, also, return them through these lockers. Through constantly changing lockerpasscodes, shipment security is maintained (Montreuil, and Faugere, 2016 $6^{[19]}$ ). 
During the testing phase, multiple features were tested. Some of the important parameters, but not limited to, that are advised to exist in the smart lockers, are :

- Constant CCTV camera monitoring

- Lan or 3G connectivity

- Parcel security measures including weight capture, door opening sensor

- As fail-safe, internal battery and option for offline information storage of customer and passcode information

- Possibility for cash-on-delivery through debit / credit card

- User friendly GUI for even amateur users

- NFC connectivity (future possibility)

Additionally, this serves as real estate for marketing and helps in increasing foot-fall in respective stores, thus a mutually beneficial scenario for all parties involved. Almost all major delivery companies across the world, including postal services, Amazon, Wall Mart, DHL, UPS etc., are investing heavily in smart lockers. In India, companies, like QikPod, Smart Box plan to have at least 50,000 lockers installed in India's 10 biggest cities. Use of Smart Lockers can reduce failed attempts, improve productivity of FEs by providing a single drop point for multiple shipments, allow users to receive shipments at their own convenience and allays their privacy concerns, and thus, reduce operational costs.

\section{Conclusion}

In this paper, we identified few of the existing inefficiencies, in the LM of any logistics, especially in Indian environments. that arise due to subjectivity of the customers and the tasks being highly labour intensive. These inefficacies can lead to shrinkages, inability to have a well-defined delivery plan, reduced customer satisfaction and trust, and reduced sales. To alleviate them, various technology solutions are explored and some case studies presented from different companies, including Flipkart.

The presented solutions are modular in nature and can work in parallel or in conjunction to bring the desired benefits. Based on their existing problems, and current status quo, supply chain companies, can pick and choose whichever solution gives most benefit. A simple heuristic approach is to pick and choose a custom solution relevant to the respective organization. For example, a nascent and relatively capital deprived company can start with only GPS and Smart Lockers as capabilities. As the strength and propensity to spend increases, the company can focus more on customer centric initiatives. Table 1 presents mapping of presented solutions to the issues that LM of a logistics company suffers.

Table 1: Solutions Summary

\begin{tabular}{lcc}
\hline Category & Problem & Possible Solutions \\
\hline \multirow{2}{*}{$\begin{array}{l}\text { Customer } \\
\text { Experience }\end{array}$} & Undelivered packages & Smart Locker \\
\cline { 2 - 3 } & Rude behaviour & Voice recorder \\
\cline { 2 - 3 } Reliability & Unprofessional attitude & Voice recorder \\
& Unprofessional grooming & Auto Grooming Check \\
\cline { 2 - 3 } & Shipment to the wrong person & Voice recorder \\
\cline { 2 - 3 } & Fake attempts and incorrect delivery data & GPS \\
\cline { 2 - 3 } & Erroneous customer address & GPS \\
\hline \multirow{2}{*}{ Safety } & Low FE productivity & GPS \\
& Accidents & GPS \\
\cline { 2 - 3 } & Theft or worse crimes & GPS \\
\hline
\end{tabular}




\section{References}

Mark Millar, "Challenges of the Last Mile Delivery in Serving E-Commerce Business", June 2015 : www.koganpage.com/article/challenges-of-the-lastmile-delivery-in-serving-e-commerce-business

www.livemint.com/Companies/Ig34ixeE1nJZAjM $\mathrm{S} 15 \mathrm{Wg} 0 \mathrm{O} / \mathrm{So}$-what-is-the-state-of-Indias-onlineretail-market-anyway.html

Alexander Krasnikov, Satish Jayachandran, \& V. Kumar. "The Impact of Customer Relationship Management Implementation on Cost and Profit Efficiencies", Evidence from the U.S. Commercial Banking Industry, 2009

Berger, Allen N., J. David Cummins, and Mary A. Weiss, "The Coexistence of Multiple Distribution Systems for Financial Services: The Case of Property Liability Insurance," Report Bo. 95-13 (1995), Financial Institutions Center, the Wharton School, University of Pennsylvania.

Kuehner-Herbert, K., 2009 “A growing defection: more clients ready to switch as trust wanes", American Banker: The Financial Services Daily.

Ind, N. (2004), Living the Brand: How to Transform Every Member of Your Organization into a Brand Ambassador, 2nd ed., Kogan Page, London.

Thompson, Ed and Michael Maoz, "What Is Hot in the World of CRM Applications," Gartner Group, Report No. G00124558 (2005).

Bolton, Ruth, "A Dynamic Model of the Duration of the Customer's Relationship with a Continuous Service Provider: The Role of Satisfaction," Marketing Science, 17 (1), 45-65 (1998).

Reinartz, Werner, Jacqueliyn S. Thomas, and V. Kumar, "Balancing Acquisition and Retention
Resources to Maximize Customer Profitability," Journal of Marketing, 69 (January, 2005), 63-79.

Johnson, Michael and Fred Selnes, "Customer Portfolio Management: Toward a Dynamic Theory of Exchange Relationships," Journal of Marketing, 68 (April, 2004), 1-17.

Cadwallader, S., Burke Jarvis, C., Bitner, M.J. and Ostrom, A.L., "Frontline employee motivation to participate in service innovation implementation", Journal of the Academy of Marketing Science, Vol. 38, pp. 219-239 (2010).

https://www.linkedin.com/pulse/dude-where-mypackage-impact-missed-deliverie-kela-ivonye

http://www.livemint.com/Companies/tLNhdsTYnn uQ1jc9FHKvqO/Blue-Dart-introduces-lockerservice-for-ecommerce-packages.html

http://www.livemint.com/Companies/afb2SZHVXi cymHZumCgm5L/Lastmile-delivery-hurdles-paveway-for-surge-in-new-startu.html

https://www.boston.com/news/crime/2017/02/17/9 0000-worth-of-diapers-stolen-from-truck-headedto-amazon-facility

https://www.bloomberg.com/news/features/201611-16/meet-the-flipkart-delivery-men-in-india-s-ecommerce-battle

http://www.dnaindia.com/india/report-bengaluruyouth-kills-flipkart-delivery-man-for-rs-12000smartphone-2283165

https://www.liveviewgps.com/blog/gps-trackingsaves-money-commercial-auto-insurance/

Louis Faugere, Benoit Montreuil, "Hyperconnected City Logistics: Smart Lockers Terminals \& Last Mile Delivery Networks", $3^{\text {rd }}$ International Physical Internet Conference, July, 2016 